\title{
Chronological spacetimes without lightlike lines are stably causal
}

\author{
E. Minguzzi \\ Dipartimento di Matematica Applicata, Università degli Studi di Firenze, Via S. Marta 3, \\ I-50139 Firenze, Italy \\ E-mail: ettore.minguzzi@unifi.it
}

\begin{abstract}
The statement of the title is proved. It implies that under physically reasonable conditions, spacetimes which are free from singularities are necessarily stably causal and hence admit a time function. Read as a singularity theorem it states that if there is some form of causality violation on spacetime then either it is the worst possible, namely violation of chronology, or there is a singularity. The analogous result: "Non-totally vicious spacetimes without lightlike rays are globally hyperbolic" is also proved, and its physical consequences are explored.
\end{abstract}

\section{Introduction}

While the local structure of spacetime is fairly simple to describe, there are still a number of open problems concerning the causal behavior of the spacetime manifold in the large. About three decades ago Geroch and Horowitz in the conclusions of their review "Global structure of spacetimes" 8] identified the problem of giving good physical reasons for assuming stable causality as one of the most important questions concerning the global aspects of general relativity together with the proof of the cosmic censorship conjecture. Indeed, if stable causality holds then the spacetime does not suffer any pathological behavior connected with the presence of almost closed causal curves, and, more importantly, it admits a (non-unique) time function [9, that is a function which is continuous and increases on every causal curve.

In order to understand the role of stable causality it is useful to recall that most conformal invariant properties can be ordered in the so called causal ladder of spacetimes (see figure 1). If the real Universe were represented by a globally hyperbolic manifold (the top of the ladder) then a number of mathematical and physical nice properties would hold. The problem is that, though there is evidence that the spacetime manifold evolves according to the Einstein equations, it is not clear whether the evolution from physically reasonable Cauchy 
data would introduce naked singularities and would eventually produce a nonglobally hyperbolic spacetime. If so, the Cauchy data would be insufficient for the determination of the spacetime geometry and one would have to take into account the information coming from infinity. However, Penrose gave arguments which support the view that the so developed manifold would actually be globally hyperbolic [23] (strong cosmic censorship).

Some other authors claim that one should only expect that the non-predictable behavior due to naked singularities be confined behind horizons (weak cosmic censorship). Other authors note that there is not even compelling reasons for excluding chronologically violating regions, in fact in some cases they allow to keep the spacetime non-singular even in presence of trapped surfaces [21. From this point of view chronology violating sets should not be discarded a priori, instead they should be considered in the same footing as naked singularities, a physical possibility which hopefully remains hidden behind an horizon. These considerations show that the class of mathematically reasonable spacetimes is considerably large, and therefore physicists look for physical arguments which allow to get as close as possible to global hyperbolicity. In short physicists look for results which allow to climb the causal ladder.

The first step would be to justify the chronology property. Actually this assumption is philosophically satisfactory because its violation would arise issues related to the free will of the generic observer. However, the notion of free will is not modeled in general relativity, therefore it becomes reasonable to search for other physical mechanisms, perhaps based on quantum mechanics, which prevent the formation or stability of chronology violating sets. The idea that such a mechanism should indeed exist and that starting from well behaved initial conditions closed timelike curves can not form has been referred by Hawking as the chronology protection conjecture 10 . As I commented above there is no general consensus on its validity and the evidence coming from classical general relativity is under investigation [29,28, 32,13 .

It is natural to separate the remainder of the causal ladder in two parts. That going from chronology up to stable causality (causality, distinction, strong causality belong to it), and that going from stable causality up to global hyperbolicity (passing through causal continuity and causal simplicity). While the former part deals with each time more demanding conditions conceived to avoid almost closed causal curves, the latter part presents each time more demanding conditions in order to reduce the effects of points at infinity on spacetime.

The problem of climbing the causal ladder from chronology up to stable causality will be considered and solved in this work. It has received less attention than the latter problem, that is, that of going from stable causality up to global hyperbolicity which is indeed more closely related to the strong cosmic censorship conjecture [23].

I am going to prove that chronology plus the absence of lightlike lines implies stable causality (theorem 6). The theorem is formulated so that every mentioned property is conformally invariant. It is therefore a theorem on the causal structure of spacetime. In this respect it is important to use the weaker assumption of absence of lightlike lines instead of the more common null convergence, genericity and completeness conditions, though these have a more direct physical meaning. In any case the requirement of absence of lightlike lines can be regarded as a null completeness assumption, that is, it follows from demanding absence of 
singularities. I shall say more on this correspondence in the first section. Thus the theorem physically can be interpreted by saying that under chronology, the absence of singularities implies stable causality and hence the existence of a time function. It is the first result of this form which reduces the existence of a time function to considerable less demanding properties. Moreover, note that in the previous statement the required absence of singularities is more precisely only a null completeness requirement: the spacetime manifold could still be timelike incomplete in a way compatible with the singularity theorems (I shall say more on that in sections 4 and 6).

Recall that stable causality is the best possible constraint in order to remove almost closed causal curves and hence causality violations. The theorem can then be regarded as a singularity theorem, indeed, rewritten in the form non-stably causal spacetimes either are non-chronological or admit lightlike lines receives the following physical interpretation if there is a form of causality violation on spacetime then either it is the worst possible, namely violation of chronology, or the spacetime is singular. Regarded in this way the theorem clarifies the influence of causality violations on singularities. In fact, if the violation of chronology is regarded as a sort of singularity then the theorem states that if there is no time function then the spacetime is singular in this broader sense.

I refer the reader to [20, 16] for most of the conventions used in this work. In particular, I denote with $(M, g)$ a $C^{r}$ spacetime (connected, time-oriented Lorentzian manifold), $r \in\{3, \ldots, \infty\}$ of arbitrary dimension $n \geq 2$ and signature $(-,+, \ldots,+)$. On $M \times M$ the usual product topology is defined. For convenience and generality I often use the causal relations on $M \times M$ in place of the more widespread point based relations $I^{+}(x), J^{+}(x), E^{+}(x)$ (and past versions). All the causal curves that we shall consider are future directed (thus also the past rays). The subset symbol $\subset$ is reflexive, $X \subset X$. Several versions of the limit curve theorem will be repeatedly used, particularly those referring to sequences of $g_{n}$-causal curves, where the metrics in the sequence $g_{n}$ may differ. The reader is referred to [15] for a sufficiently strong formulation.

\section{Absence of lightlike lines}

In this section I consider the property of absence of lightlike lines and comment on its physical meaning.

Two spacetimes belonging to the same conformal class $(M, \boldsymbol{g})$ share the same lightlike geodesics up to reparametrizations, and the condition of maximality for the lightlike geodesic $\gamma$ reads "there is no pair of events $x, z \in \gamma,(x, z) \in I^{+}$", which makes no mention to the full metric structure and hence is independent of the representative of the conformal class. Thus, it is convenient to give the following conformal invariant definition,

Definition 1. A lightlike line is an achronal inextendible causal curve.

The definition implies, by achronality, that the causal curve is a lightlike geodesic and that it maximizes the Lorentzian length between any of its points.

It is well known that [22, Chap. 10, Prop. 48]

Proposition 1. If a inextendible lightlike geodesic admits a pair of conjugate events then it is not a lightlike line. 
It can be proved that the notion of conjugate points along a lightlike geodesic is conformally invariant [20], thus the previous proposition relates two conformal invariant properties. In particular note that the requirement every lightlike geodesic has a pair of conjugate points is stronger than absence of lightlike lines, e.g. $1+1$ Minkowski spacetime with $x=0$ and $x=1$ identified. From the point of view of Lorentzian geometry any statement should be formulated so as to make its conformal invariance clear. For physical reasons some authors prefer to mention physically motivated but non-conformal invariant conditions. The consequence, however, is that several results have been formulated in an unnecessary weak form as the assumptions of the theorems are not really used.

Definition 2. An inextendible lightlike geodesic $\gamma$ of the spacetime $(M, g)$ satisfies the generic condition if at some $x \in \gamma$ the tangent vector $n$ to the curve is a generic vector, that is, $n^{c} n^{d} n_{[a} R_{b] c d\left[e n_{f]}\right.} \neq 0$. A spacetime satisfies the null generic condition if every inextendible lightlike geodesic satisfies the generic condition.

A spacetime can be generic only if $n \geq 3$ (see [3, Cor. 2.10]). The precise sense in which the null generic condition is generic is clarified by [3, Prop. 2.15]. It is usually assumed on the physical ground that if a lightlike geodesic does not satisfy it then arbitrarily small metric perturbation in the geodesic path would make it true.

Definition 3. The spacetime $(M, g)$ satisfies the timelike convergence condition if $R(v, v) \geq 0$ for all timelike, and hence also for all lightlike, vectors $v$. The spacetime $(M, g)$ satisfies the null convergence condition if $R(v, v) \geq 0$ for all lightlike vectors $v$ (cf. [11, p.95] [3, Def. 12.8]).

Thus the null convergence condition is a consequence of the positivity of the energy density.

Definition 4. A spacetime $(M, g)$ is null geodesically complete if every inextendible lightlike geodesic is complete.

Proposition 2. In a spacetime $(M, g)$ of dimension $\operatorname{dim} M \geq 3$, which satisfies the null convergence condition, the null generic condition and that is null geodesically complete every inextendible lightlike geodesic admits a pair of conjugate events. In particular $(M, g)$ does not have lightlike lines.

Proof. It follows from the existence of some pair of conjugate points in the lightlike geodesics accordingly to [11, Prop. 4.4.5] [3, Prop. 12.17].

This proposition has been improved by Tipler [30,31] and Chicone and Ehrlich 6] (see also Borde 5]) by weakening the null convergence condition to the averaged null convergence condition. This possibility is important because many quantum fields on spacetime determine a stress-energy tensor and hence a Ricci tensor which does not comply with the null convergence condition while it satisfies the averaged null convergence condition.

Proposition 2 implies that the condition of absence of lightlike lines is quite reasonable from a physical point of view at least if the spacetime is assumed to be non-singular (see also the discussion in [11, Sect. 4.4]) or just null geodesically complete. 
In the next sections I will prove that the assumption of absence of lightlike lines has the effect of identifying the levels of the causal ladder between chronology and stable causality. In this respect the hard part will come with the inclusion of stable causality. A key role will be played by the property of $K$-causality introduced by Sorkin and Woolgar [27, and for the last step by a new property which I study in the next section.

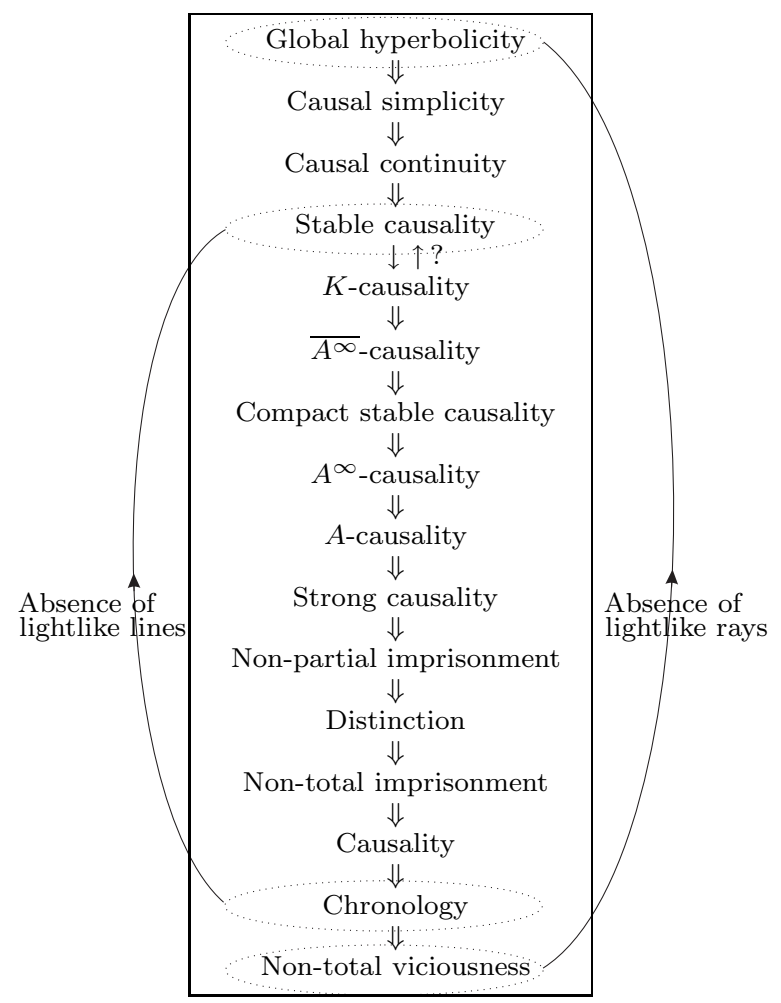

Fig. 1. The causal ladder displaying the new levels considered in section 3 Penrose's infinite ladder between $A$-causality and $A^{\infty}$-causality is omitted [16], as well as the levels of weak distinction and feeble distinction [19. For the placement of the non-imprisonment properties the reader is referred to [18. The arrow $C \Rightarrow D$ means that $C$ implies $D$ and there are examples which show that $C$ differs from $D$. Stable causality implies $K$-causality but it is not known if they coincide. The implications climbing the ladder express the geometrical content of the theorems proved in this work. 


\section{Compact stable causality}

Recall that a non-total imprisoning spacetime is a spacetime for which there is no future-inextendible causal curve totally imprisoned in a compact (future non-total imprisonment is equivalent to past non-total imprisonment [2,18]). It is known that every relatively compact open set in a non-total imprisoning spacetime [18] is stably causal when regarded as a spacetime with the induced metric [2]. Actually, this property characterizes non-total imprisonment, indeed we have

Theorem 1. A spacetime $(M, g)$ is non-total imprisoning iff for every relatively compact open set $B,\left(B,\left.g\right|_{B}\right)$ is stably causal.

Proof. The implication to the right was proved by Beem [2]. To the left, assume $(M, g)$ has a compact $C$ in which some curve $\gamma$ is future imprisoned. In [18] I proved that there is a lightlike line $\eta$ contained in $C$ such that $\eta \subset \Omega_{f}(\eta)$ where $\Omega_{f}(\eta)$ is the set of accumulation points in the future of $\eta$ (in analogy with the set of $\omega$-limit points of dynamical systems). Let $B$ be a relatively compact open set such that $C \subset B$. Take $q \in \eta$ and, given a convex neighborhood $U \ni q$, $U \subset B$, take $p \in \eta \cap J_{\left(U,\left.g\right|_{U}\right)}^{-}(q)$. Take $g^{\prime}>g$ in $B$ ( $g^{\prime}$ need not be defined on $\left.B^{C}\right)$ then $p \in I_{\left(U, g^{\prime}\right)}^{-}(q)$, but recall that $p \in \Omega_{f}(\eta)$ is an accumulation point for the future-inextendible $g^{\prime}$-timelike curve given by the portion of $\eta$ which starts from $q$. Thus since $I_{\left(U,\left.g^{\prime}\right|_{U}\right)}^{-}$is open it is possible to construct a closed $g^{\prime}$-timelike curve contained in $B$. The argument holds for any choice of $g^{\prime}$ thus it is not true that for every relatively compact open set $B,\left(B,\left.g\right|_{B}\right)$ is stably causal.

Note that non-total imprisonment is a quite weak property (it is implied by weak distinction [18]). A related problem is that of establishing if, given an arbitrary compact on spacetime, the metric can be widened in it without introducing closed causal curves in the whole spacetime. If this is possible the spacetime satisfies a condition which is stronger than non-total imprisonment. We can define a new property

Definition 5. A spacetime $(M, g)$ is compactly stably causal if for every relatively compact open set $B$ there is a metric $g_{B} \geq g$ such that $g_{B}>g$ on $B$, $g_{B}=g$ on $B^{C}$ and $\left(M, g_{B}\right)$ is causal.

Remark 1. There are some equivalent definitions, for instance: $(M, g)$ is compactly stably causal if for every compact set $C$ there is $g_{C} \geq g$ such that $g_{C}>g$ on $C$ and $\left(M, g_{C}\right)$ is causal. In order to prove the equivalence one has to take appropriate convex combinations of metrics with smooth coefficients.

Some natural questions arise, among them the placement of compact stable causality in the causal ladder of spacetimes. Before considering this question let me recall some notation and terminology [16]. Following Woodhouse [33, 1. I denote with $A^{+}$the closure of the causal relation, that is $A^{+}=\bar{J}^{+}$. A spacetime is $A^{\infty}$-causal if there is no finite cyclic chain of distinct $A^{+}$-related events. This property is equivalent to the antisymmetry of the relation $A^{+\infty}=$ $\cup_{i=1}^{+\infty}\left(A^{+}\right)^{i}$, which is the smallest transitive relation containing $A^{+}$. Analogously, a spacetime is $\overline{A^{\infty}}$-causal if the relation $\overline{A^{+\infty}}$ is antisymmetric. The relation $K^{+}$ is the smallest closed and transitive relation containing $\mathrm{J}^{+}$, and the spacetime 
is $K$-causal if the relation $K^{+}$is antisymmetric [27]. It is known that stable causality implies $K$-causality, although it is not known if these two conditions coincide [17]. We have

Theorem 2. K-causality implies $\overline{A^{\infty}}$-causality.

Proof. Since $J^{+} \subset K^{+}$, any causal relation obtained from $J^{+}$by taking closures or by making the relation transitive through the replacement $R^{+} \rightarrow \cup_{i=0}^{+\infty}\left(R^{+}\right)^{i}$, is still contained in $K^{+}$. Since $\overline{A^{+\infty}}$ has this form $\overline{A^{+\infty}} \subset K^{+}$, thus $K$-causality implies $\overline{A^{\infty}}$-causality.

Remark 2. Given a relation $R^{+}$the two involutive operations given by (a) closure: $R^{+} \rightarrow \bar{R}^{+}$, and (b) transitivization: $R^{+} \rightarrow R^{+\infty}=\cup_{i=1}^{+\infty}\left(R^{+}\right)^{i}$, once alternatively applied to $J^{+}$generate a chain of relations all contained in $K^{+}$ whose first members are $J^{+}, A^{+}, A^{+\infty}, \overline{A^{+\infty}}, \cdots$. By demanding the antisymmetry one obtains a ladder of causal properties whose first members are causality, $A$-causality, $A^{\infty}$-causality and $\overline{A^{\infty}}$-causality, all necessarily weaker than $K$ causality. If at a certain point two adjacent relations coincide then they coincide with $K^{+}$as they are both closed an transitive and they are certainly the smallest relations with this property. In this case the mentioned ladder of relations finishes there where this coincidence occurs. As we shall see, the mentioned first levels are all different but it is not known if from some point on the levels would start to coincide, that is, if after a finite number of operations of closure and transitivization one would get $K^{+}$and $K$-causality. Examples support the view that this coincidence occurs at a level which increases with the dimensionality of the spacetime.

Lemma 1. Let $\circ$ denote the composition of relations, then $J^{+} \circ A^{+} \subset A^{+}$and $A^{+} \circ J^{+} \subset A^{+}$.

Proof. Let us consider the former case, the latter being analogous. Let $(x, y) \in$ $J^{+}$and $(y, z) \in A^{+}$, and let $\gamma_{n}$ be a sequence of causal curves of endpoints $\left(y_{n}, z_{n}\right) \rightarrow(y, z)$. Take $x_{k} \in I^{-}(x), x_{k} \rightarrow x$, so that $x_{k} \ll y$ and for sufficiently large $n, x_{k} \ll y_{n} \leq z_{n}$, thus $\left(x_{k}, z_{n(k)}\right) \in I^{+}$and in the $\operatorname{limit}(x, z) \in A^{+}$.

Theorem 3. $\bar{A}^{\infty}$-causality implies compact stable causality.

Proof. Suppose $(M, g)$ is $\overline{A^{\infty}}$-causal but non-compactly stably causal, then there is a relatively compact open set $B$ such that for every $g^{\prime} \geq g, g^{\prime}>g$ on $B, g^{\prime}=g$ on $B^{C},\left(M, g^{\prime}\right)$ is not causal. Let $g_{n}$ be a sequence of metrics $g_{n} \geq g, g_{n}>g$ on $B, g_{n}=g$ on $B^{C}, g_{n+1} \leq g_{n}$, and $g_{n} \rightarrow g$ pointwisely on the appropriate tensor bundle. For every choice of $n,\left(M, g_{n}\right)$ is not causal, and since $(M, g)$ is causal there must be a closed $g_{n}$-causal curve $\gamma_{n}$ intersecting $B$ (see figure2). Let $p_{n}^{0} \in \gamma_{n} \cap B$ and parametrize the curves with respect to a complete Riemannian metric $h$ so that $p_{n}^{0}=\gamma_{n}(0)$.

Assume an infinite number of $\gamma_{n}$ is entirely contained in $\bar{B}$. Beem [2] has shown that there would be a inextendible $g$-causal limit curve contained in $\bar{B}$ in contradiction with the non-total imprisoning property of the spacetime (recall that $A$-causality implies distinction which implies the non-total imprisoning property). Thus without loss of generality we can assume that none of the $\gamma_{n}$ is 
entirely contained in $\bar{B}$. We conclude that $\gamma_{n}$ intersects $\dot{B}$ at least once to enter $B^{C}$. Without loss of generality we can also assume that $p_{n}^{0} \rightarrow p^{0} \in \bar{B}$.

Using again the limit curve argument, through $p^{0}$ there passes a future inextendible (hence its $h$-length parameter has domain $(-\infty,+\infty)) g$-causal curve $\gamma^{0}$ which can't pass through $p^{0}$ twice as it would imply a violation of causality for $(M, g)$. In particular since $(M, g)$ is non-partial imprisoning it escapes $\bar{B}$ at a last point $q^{0} \in \dot{B}$ to never reenter $\bar{B}$. Let $\gamma_{n}^{0}$ be a subsequence of $\gamma_{n}$ which converges to $\gamma^{0}$ uniformly on compact subsets and let $s^{0}$ be the value of the parameter such that $q^{0}=\gamma^{0}\left(s^{0}\right)$. Since $\gamma_{n}^{0}\left(s^{0}+2\right) \rightarrow \gamma^{0}\left(s^{0}+2\right) \notin \bar{B}$ pass to a subsequence denoted in the same way so that $\gamma_{n}^{0}\left(s^{0}+2\right) \notin \bar{B}$. Let $\left(\bar{s}_{n}^{0}, t_{n}^{1}\right) \ni s^{0}+2$ be the largest open connected interval so that $\gamma_{n}^{0}\left(\left(\bar{s}_{n}^{0}, t_{n}^{1}\right)\right) \subset(\bar{B})^{C}$. Define $\bar{q}_{n}^{0}, p_{n}^{1} \in \dot{B}$ as $\bar{q}_{n}^{0}=\gamma_{n}^{0}\left(\bar{s}_{n}^{0}\right)$ and $p_{n}^{1}=\gamma_{n}^{0}\left(t_{n}^{1}\right)$. Let $p^{1} \in \dot{B}$ be an accumulation point for $p_{n}^{1}$, without loss of generality we can assume $p_{n}^{1} \rightarrow p^{1}$. Note that the segment $\left.\gamma_{n}^{0}\right|_{\left[s_{n}^{0}, t_{1}^{n}\right]}$ is entirely contained in $B^{C}$ and hence it is $g$-causal. Since $\bar{s}_{n}^{0} \in\left[0, s^{0}+2\right]$, without loss of generality we can assume $\bar{s}_{n}^{0} \rightarrow \bar{s}^{0}$ for some $\bar{s}^{0}$. Now, $\bar{s}^{0} \leq s^{0}$ indeed if $\bar{s}^{0}>s^{0}$ then $\bar{q}_{n}^{0} \in \bar{B}$ converges to $\gamma^{0}\left(\bar{s}^{0}\right)$ a point that does not belong to $\bar{B}$ which is impossible. In particular, it is possible to find a sequence $s_{n}^{0}$, $\bar{s}_{n}^{0}<s_{n}^{0}<s^{0}+2$, such that $s_{n}^{0} \rightarrow s^{0}$. Then $q_{n}^{0}=\gamma_{n}^{0}\left(s_{n}^{0}\right) \notin \bar{B}$ converges to $q^{0}$ and the $g$-causal sequence of curves $\left.\gamma_{n}^{0}\right|_{\left[s_{n}^{0}, t_{1}^{n}\right]}$ has endpoints $\left(q_{n}^{0}, p_{n}^{1}\right) \in J^{+}$such that $\left(q_{n}^{0}, p_{n}^{1}\right) \rightarrow\left(q^{0}, p^{1}\right)$, i.e. $\left(q^{0}, p^{1}\right) \in A^{+}$. Note that $\left(p^{0}, q^{0}\right) \in J^{+}$as both points belong to $\gamma^{0}$, hence $\left(p^{0}, p^{1}\right) \in A^{+}$.

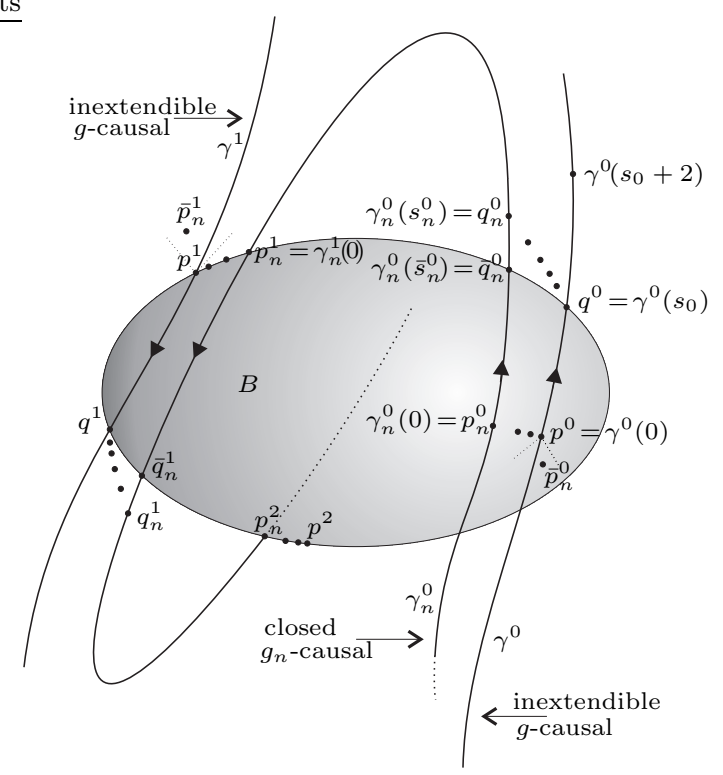

Fig. 2. The argument of the proof that $\overline{A^{\infty}}$-causality implies compact stable causality.

The limit curve theorem states that $t_{n}^{1} \rightarrow+\infty$ otherwise $p^{1}$ would belong to the prolongation of $\gamma^{0}$ which is impossible since $q^{0}$ is the last point of $\gamma^{0}$ in $\bar{B}$. The segments $\left.\gamma_{n}^{0}\right|_{\left[\bar{s}_{n}^{0}, t_{1}^{n}\right]}$ are not all contained in a compact because $\gamma^{0}$ escapes every 
compact to never return and for every $k>0, \gamma_{n}^{0}\left(s_{n}^{0}+k\right) \rightarrow \gamma^{0}\left(s^{0}+k\right)$ because $t_{n}^{1} \rightarrow+\infty$. As a consequence the pair $\left(p^{0}, p^{1}\right) \in A^{+}$can be regarded as the limit of the pairs of endpoints of $g$-causal segments which are not all contained in a compact. (In order to construct these segments take $\bar{p}_{k}^{0} \in I^{-}\left(p^{0}\right), \bar{p}_{k}^{0} \rightarrow p^{0}$ so that $q^{0} \in I^{+}\left(\bar{p}_{k}^{0}\right)$ and hence since $I^{+}$is open $q_{n(k)}^{0} \in I^{+}\left(\bar{p}_{k}^{0}\right)$ for a sufficiently large $n(k)$. Next follow the $g$-causal segment $\left.\gamma_{n}^{0}\right|_{\left[s_{n}^{0}, t_{1}^{n}\right]}$ which are not all contained in a compact, finally redefine the parametrization of the sequence $\bar{p}_{k}^{0}$ and pass if necessary to a subsequence so that $\left(\bar{p}_{n}^{0}, q_{n}^{0}\right) \in I^{+}$and hence $\left(\bar{p}_{n}^{0}, p_{n}^{1}\right) \in J^{+}$ with $\left(\bar{p}_{n}^{0}, p_{n}^{1}\right) \rightarrow\left(p^{0}, p^{1}\right)$.) In particular, $p^{0} \neq p^{1}$ since the spacetime is strongly causal.

Now, translate all the parametrizations of $\gamma_{n}^{0}$ so that $t_{n}^{1}$ gets replaced by 0 . Repeat the previous steps where now $p^{1}$ plays the role of $p^{0}$ and the found sequence $\gamma_{n}^{1}$ is a reparametrized subsequence of $\gamma_{n}^{0}$.

Continue in this way, defining for at each step analogous subsequences and events so that $p^{k} \in \bar{B},\left(p^{k}, p^{k+1}\right) \in A^{+}, p^{k} \neq p^{k+1}$, and for each $k$ there is a sequence of $g$-causal curves, not all contained in a compact, so that the endpoints of the sequence converge to $\left(p^{k}, p^{k+1}\right)$. Note that for every pair of positive integers $a \leq b,\left(p^{a}, p^{b}\right) \in A^{+\infty}$.

Since $\bar{B} \times \bar{B}$ is compact, there is a subsequence denoted $\left(p^{k_{s}}, p^{k_{s}+1}\right)$ such that $\left(p^{k_{s}}, p^{k_{s}+1}\right) \rightarrow(x, z)$ as $s \rightarrow+\infty$. Moreover, $x \neq z$ because otherwise for every relatively compact causally convex neighborhood $U \ni x$, for sufficiently large $s,\left(p^{k_{s}}, p^{k_{s}+1}\right) \in U$, and the sequence of $g$-causal curves not all contained in a compact, whose endpoints converge to $\left(p^{k_{s}}, p^{k_{s}+1}\right)$ would contradict the causal convexity of $U$. Since $A^{+}$is closed, $(x, z) \in A^{+}$and $x \neq z$. Since $p^{k_{s}}$ is a subsequence of $p^{k}$, for every $s, k_{s}+1 \leq k_{s+1}$, thus $\left(p^{k_{s}+1}, p^{k_{s+1}}\right) \in A^{+\infty}$ and in the limit $s \rightarrow+\infty,(z, x) \in \overline{A^{+\infty}}$. As a consequence $(M, g)$ is not $\bar{A}^{\infty}$-causal which is the searched contradiction.

Theorem 4. Compact stable causality implies $A^{\infty}$-causality.

Proof. Assume the spacetime is compactly stably causal, and suppose it is not $A^{\infty}$-causal then there is a finite closed chain of $A^{+}$-related events $\left(x_{i}, x_{i+1}\right) \in$ $A^{+}, i=1, \ldots, n, x_{n+1}=x_{1}$.

Consider a relatively compact open set $B$ which contains all $x_{i}, i=1, \ldots, n$, and let $g_{B} \geq g, g_{B}>g$ on $B, g_{B}=g$ on $B^{C}$. We want to prove that $A^{+} \cap$ $(B \times B) \subset J_{\left(M, g_{B}\right)}^{+}$, from which it follows that $\left(M, g_{B}\right)$ is not causal whatever the choice of $g_{B}$ and hence $(M, g)$ is not compactly stably causal, the searched contradiction. Let $(y, z) \in A^{+}, y, z \in B$, then by the limit curve theorem either $(y, z) \in J^{+} \subset J_{\left(M, g_{B}\right)}^{+}$or there are a future inextendible $g$-causal curve $\sigma^{y}$ starting from $y$, and a past inextendible $g$-causal curve $\sigma^{z}$ ending at $z$ such that for every $y^{\prime} \in \sigma^{y} \backslash\{y\}$ and $z^{\prime} \in \sigma^{z} \backslash\{z\},\left(y^{\prime}, z^{\prime}\right) \in A^{+}$. At least a segment of $\sigma^{y}$ near $y$ is timelike for $\left(M, g_{B}\right)$ and analogously for $\sigma^{z}$, thus $\left(y, y^{\prime}\right) \in I_{\left(M, g_{B}\right)}^{+}$, and $\left(z^{\prime}, z\right) \in I_{\left(M, g_{B}\right)}^{+}$finally since $\left(y^{\prime}, z^{\prime}\right) \in A^{+} \subset \overline{J_{\left(M, g_{B}\right)}^{+}}$, it is $(y, z) \in I_{\left(M, g_{B}\right)}^{+}$.

Remark 3. All the properties of the previous theorems differ. In [16] I gave an example of non- $K$-causal $A^{\infty}$-causal spacetime. A closer inspection proves that it is actually non- $\bar{A}^{\infty}$-causal but compactly stably causal. Moreover, it is possible to construct an example, similar to that of [16] which is $\overline{A^{\infty}}$-causal but non- $K$ causal (simply repeat the figure of [16] three times vertically, and then identify 
the holes cyclically). The properties $A^{\infty}$-causality and compact stable causality differ because of the spacetime example of figure 3 A consequence of these examples is the perhaps surprising fact that compact stable causality differs from stable causality (see again the example of [16). This fact means that the behavior of the light cones near infinity is important in order to determine if a spacetime is properly compactly stably causal or not.

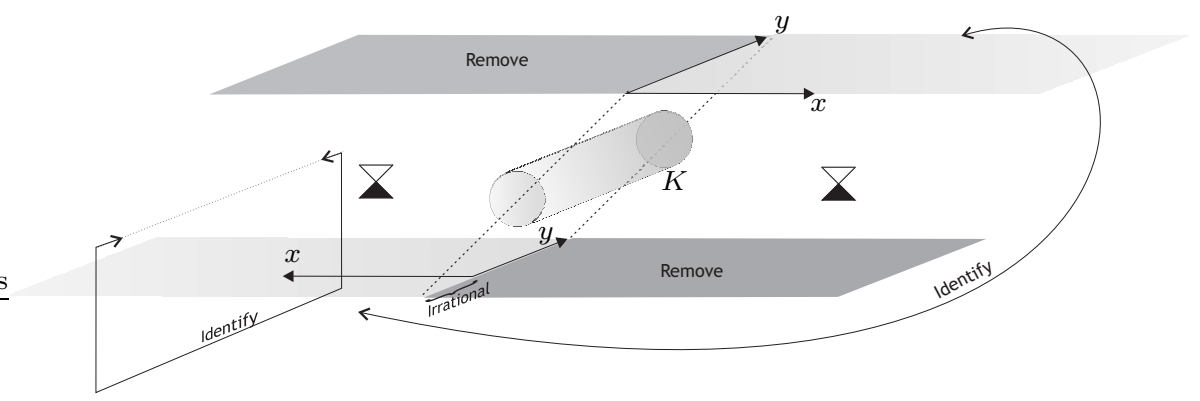

Fig. 3. A $A^{\infty}$-causal but non-compactly stably causal spacetime. In order to construct the spacetime start from $\mathbb{R} \times S^{1} \times \mathbb{R}$ of coordinates $(t, \theta, z), \theta \in[0,1]$, and metric $g=$ $-\mathrm{d} t^{2}+\mathrm{d} \theta^{2}+\mathrm{d} z^{2}$, remove two spacelike surfaces and identify, after a translation by an irrational number, two spacelike surfaces as done in the figure. The coordinates $(x, y)$ have been introduced on the identified surfaces so as to make the identification clear. The spacetime is non-orientable but this feature is not essential. The spacetime is non-compactly stably causal since any enlargement of the metric on $K$ gives closed causal curves. Thanks to the translation by an irrational number there cannot be closed chains of $A^{+}$related events.

\section{The proof and some physical considerations}

I start with a result due to Hawking [12] 11, Prop. 6.4.6] (he proved it with the stronger but inessential assumption that every lightlike geodesic admits a pair of conjugate points)

Lemma 2. A chronological spacetime without lightlike lines is strongly causal.

Proof. Recall that in a strongly causal spacetime, given any neighborhood $U$ of $x \in M$ there exist a neighborhood $V \subset U, x \in V$, such that any future-directed causal curve with endpoints at $V$ is entirely contained in $U$ (see for instance [20, Lemma 3.22]). Thus if $(M, g)$ were not strongly causal there would be a point $x$, a neighborhood $U \ni x$, and a sequence of causal curves $\gamma_{n}$ of starting event $x_{n}$, ending event $z_{n}$ such that $x_{n} \rightarrow x, z_{n} \rightarrow x$, and the curves $\gamma_{n}$ are not entirely contained in $U$. Hence there are the conditions required by the limit curve theorem [15, theorem 3.1] case (2) which implies the existence of a lightlike line passing through $x$, a contradiction.

A fundamental step in the proof is

Theorem 5. If a spacetime does not have lightlike lines then the relation $A^{+}=$ $\bar{J}^{+}$is transitive, that is $K^{+}=A^{+}$. Moreover, if the spacetime is also chronolog$i$ cal then the spacetime is $K$-causal. 
Proof. Let us prove the transitivity of $A^{+}$. Take two pairs $(x, y) \in A^{+}$and $(y, z) \in A^{+}$and two sequences of causal curves $\sigma_{n}$ of endpoints $\left(x_{n}, y_{n}\right) \rightarrow(x, y)$, and $\gamma_{n}$ of endpoints $\left(y_{n}^{\prime}, z_{n}\right) \rightarrow(y, z)$. Apply the limit curve theorem [15] to both sequences, and consider first the case in which the limit curve in both cases does not connect the limit points. By the limit curve theorem, $\sigma_{n}$ has a limit curve $\sigma$ which is a past inextendible causal curve ending at $y$. Analogously $\gamma_{n}$ has a limit curve $\gamma$ which is a future inextendible causal curve starting from $y$. The inextendible curve $\gamma \circ \sigma$ cannot be a lightlike line thus there are points $x^{\prime} \in \sigma \backslash\{y\}$, $z^{\prime} \in \gamma \backslash\{y\}$ such that $\left(x^{\prime}, z^{\prime}\right) \in I^{+}$and (pass to a subsequence) points $x_{n}^{\prime} \in \sigma_{n}$, $x_{n}^{\prime} \rightarrow x^{\prime}$ and $z_{n}^{\prime} \in \gamma_{n}, z_{n}^{\prime} \rightarrow z^{\prime}$, thus, since $I^{+}$is open, for sufficiently large $n$, $\left(x_{n}, z_{n}\right) \in I^{+}$and finally $(x, z) \in \bar{I}^{+}=A^{+}$.

If both limit curves join the limit points then clearly $(x, z) \in J^{+} \subset A^{+}$. If, say, $\sigma$ joins $x$ to $y$ but $\gamma$ does not join $y$ to $z$, take $x_{n}^{\prime} \in I^{-}(x), x_{n}^{\prime} \rightarrow x$, so that $x_{n}^{\prime} \ll y$ and for large $n, x_{n}^{\prime} \ll y_{n}^{\prime} \leq z_{n}$, thus in the limit $(x, z) \in A^{+}$. The remaining case is analogous. Thus $A^{+}$is closed and transitive hence $A^{+}=K^{+}$.

Assume $(M, g)$ is chronological then by lemma $2(M, g)$ is strongly causal. The relation $A^{+}$is antisymmetric indeed let $(x, y) \in A^{+}$and $(y, x) \in A^{+}, x \neq y$, and let $\sigma_{n}$ of endpoints $\left(x_{n}, y_{n}\right)$ and $\gamma_{n}$ of endpoints $\left(y_{n}^{\prime}, z_{n}\right)$ be sequences of causal curves whose endpoints converge to the initial pairs $\left(x_{n}, y_{n}\right) \rightarrow(x, y)$, $\left(y_{n}^{\prime}, z_{n}\right) \rightarrow(y, x)$. Then we repeat the argument used above, that is we apply the limit curve theorem to the accumulation point $y$. Call $\sigma$ the limit causal curve for $\sigma_{n}$ and analogously let $\gamma$ be the limit causal curve for $\gamma_{n}$. If $\sigma$ connects $x$ to $y$ and $\gamma$ connects $y$ to $x$ then there is a closed causal curve on spacetime a contradiction. Let $U \ni x, V \ni y$ be two disjoint causally convex neighborhoods. If $\sigma$ connects $x$ to $y$ but $\gamma$ does not connect $y$ to $x$, then it is possible to argue as above, i.e. take $x_{k}^{\prime} \in I^{-}(x), x_{k}^{\prime} \rightarrow x$, then for sufficiently large $n$, which we can choose so that $n(k)>k, y_{n(k)}^{\prime} \in I^{+}\left(x_{k}^{\prime}\right) \cap V$, from which it follows that there is a sequence of causal curves of endpoints $x_{k}^{\prime}, z_{n(k)}$, intersecting $V$. But $\left(x_{k}^{\prime}, z_{n(k)}\right) \rightarrow(x, x)$ thus strong causality is violated at $x$. The case in which $\gamma$ connects $y$ to $x$ is analogous. The remaining case is that in which $\sigma$ is past-inextendible and $\gamma$ is future-inextendible. Then $\gamma \circ \sigma$ is a inextendible causal curve which by assumption is not a lightlike line. Moreover, since strong causality holds, this curve is not partially imprisoned in any compact, thus using the same argument as above (i.e. taking advantage of the chronality of $\gamma \circ \sigma$ ) it follows that there is a sequence of causal curves of endpoints $x_{n}, z_{n}$ not all contained in a compact. Again there is a contradiction with the strong casuality at $x$.

Clearly, if we could prove that $K$-causality is equivalent to stable causality then the main theorem would follow. Unfortunately, though there is evidence for this coincidence [17] no proof has yet been given. In fact Seifert [24, even before the introduction of $K$-causality, gave an argument which would have implied the equivalence. Unfortunately, he only sketched the proof and a recent more detailed study [17] has shown that those arguments were inconclusive. If the two causal properties are indeed equivalent it is probable that the proof would be rather involved because the $K^{+}$relation is not as easy to handle as the other causal relations. Fortunately, however, it is possible to circumvent this difficulty, and avoid a direct proof of the equivalence between stable causality and $K$ causality, by working on compact stable causality. Indeed, the previous result will be used in the following weaker form 
Corollary 1. A chronological spacetime without lightlike lines is compactly stably causal.

Now, the idea is to consider the property " $(M, g)$ is compactly stably causal and does not admit lightlike lines" to show that it is inductive (see lemma 4), that is, invariant under enlargement of the light cones over compact sets. Then it is possible to enlarge the light cones in a sequence of compact sets that cover $M$ so as to obtain a causal spacetime with strictly larger light cones (theorem 6).

Lemma 3. On $(M, g)$ let $B$ be a relatively compact open set, let $g_{n}$ be a sequence of metrics $g_{n} \geq g, g_{n}>g$ on $B, g_{n}=g$ on $B^{C}, g_{n+1} \leq g_{n}$, and $g_{n} \rightarrow g$ pointwisely on the appropriate tensor bundle. If $(M, g)$ does not have lightlike lines then all but a finite number of $\left(M, g_{n}\right)$ do not have lightlike lines.

Proof. If not we can, passing to a subsequence, assume that all $\left(M, g_{n}\right)$ have lightlike lines. Denote $\gamma_{n}$ a respective sequence of lightlike lines and assume there is one, say $\gamma_{\bar{n}}$, which does not intersect $B$. Since $g_{\bar{n}}$ and $g$ coincide outside $B, \gamma_{\bar{n}}$ is a $g$-causal curve. Also it is $g$-achronal because if there are two points $p, q \in \gamma_{\bar{n}}$ such that $(p, q) \in I_{g}^{+}$then as $g \leq g_{\bar{n}},(p, q) \in I_{g_{n}}^{+}$which is impossible because $\gamma_{\bar{n}}$ is a lightlike line on $\left(M, \bar{g}_{n}\right)$. But $\gamma_{\bar{n}}$ cannot be $g$-achronal as it would be a lightlike line of $(M, g)$, thus the overall contradiction proves that all $\gamma_{n}$ intersect $B$. Without loss of generality we can assume (pass to a subsequence if necessary) that there are $x_{n} \in B \cap \gamma_{n}$, and $x \in \bar{B}$ such that $x_{n} \rightarrow x$. By the limit curve theorem there is a inextendible $g$-causal curve $\eta$ passing through $x$. If $\eta$ is not $g$-achronal there are $y, z \in \eta$ such that $(y, z) \in I_{g}^{+} \subset I_{g_{n}}^{+}$for every $n$. But since $y$ and $z$ are limit points of the sequence $\gamma_{n}$ and $I_{g}^{+}\left(\subset I_{g_{n}}^{+}\right)$is open some of the curves $\gamma_{n}$ are not lightlike lines. The contradiction proves that $\eta$ is not only $g$-causal but also $g$-achronal thus it is a lightlike line. Again this is impossible thus the assumption that an infinite number of $\left(M, g_{n}\right)$ does admit lightlike lines has lead to a contradiction.

Lemma 4. If $(M, g)$ is compactly stably causal and without lightlike lines then for every open set of compact closure $B$ it is possible to find a metric $g_{B} \geq g$ such that $g_{B}>g$ on $B, g_{B}=g$ outside $B$, and $\left(M, g_{B}\right)$ is compactly stably causal and without lightlike lines.

Proof. Since $(M, g)$ is compactly stably causal we can find $\tilde{g}_{B}$ such that $\tilde{g}_{B}>g$ on $B, \tilde{g}_{B}=g$ outside $B$ and $\left(M, \tilde{g}_{B}\right)$ is causal. Define $g_{n}=\left(1-\frac{1}{n}\right) g+\frac{1}{n} \tilde{g}_{B}$ so that $g \leq g_{n} \leq \tilde{g}_{B}$ satisfies the assumptions of the previous lemma. Thus there is a certain element of the sequence, denote it $g_{B}$, such that $\left(M, g_{B}\right)$ does not have lightlike lines and since $g_{B} \leq \tilde{g}_{B},\left(M, g_{B}\right)$ is causal. But every causal spacetime without lightlike lines is compactly stably causal thus the thesis.

Theorem 6. If $(M, g)$ is chronological and without lightlike lines then it is stably causal.

Proof. Let $h$ be an auxiliary complete Riemannian metric, $x_{0} \in M$, and let $B_{k}=B\left(x_{0}, k\right)$ be the open balls of radius $k$ centered at $x_{0}$. Define $g_{1}=g$. By the previous lemma it is possible to find a metric $g_{2}>g_{1}$ on $B_{2}, g_{2}=g_{1}$ outside $B_{2}$, such that $\left(M, g_{2}\right)$ is compactly stably causal and without lightlike lines. Next 
repeat the argument for the relatively compact open set $B_{3}$ with respect to the spacetime $\left(M, g_{2}\right)$ : there is a metric $g_{3}>g_{2}$ on $B_{3}, g_{3}=g_{2}(=g)$ outside $B_{3}$, such that $\left(M, g_{3}\right)$ is compactly stably causal and without lightlike lines. Continue in this way and find a sequence of metrics $g_{k+1} \geq g_{k} \geq g, g_{k+1}>g_{k}$ on $B_{k+1}$. The open sets $A_{1}=B_{2}, A_{k}=B_{k+1} \backslash \bar{B}_{k-1}$ for $k \geq 2$, cover $M$. Let $\left\{\chi_{k}\right\}$ be a partition of unity so that the support of $\chi_{k}$ is contained in $A_{k}$, and define $\tilde{g}=\sum_{k=1}^{+\infty} \chi_{k} g_{k+2}$ (the sum has at most two non vanishing terms at each point) then $\tilde{g}>g$, moreover at $x \in B_{k}, \tilde{g}(x) \leq g_{k+2}(x)$, because for $n>k, \chi_{n}(x)=0$ (see figure 4). But $(M, \tilde{g})$ is causal because otherwise there is a closed $\tilde{g}$-causal curve $\sigma$, which being a closed set, is entirely contained in $B_{s}$ for some $s$. Since $\tilde{g} \leq g_{s+2}$ on $B_{s}$, this curve is $g_{s+2}$-causal which contradicts the (compact stable) causality of $\left(M, g_{s+2}\right)$. Thus since $(M, \tilde{g})$ is causal and $\tilde{g}>g,(M, g)$ is stably causal.

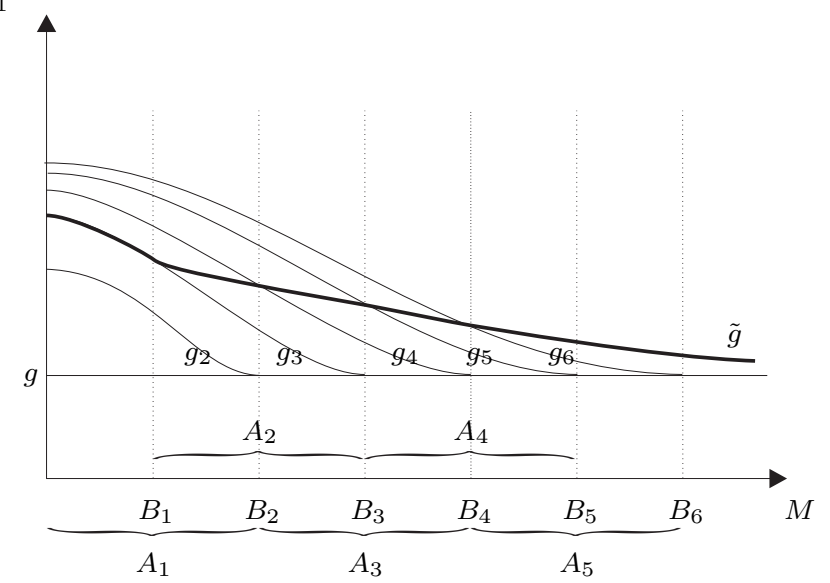

Fig. 4. The construction of the metric $\tilde{g}>g$ and of the causal spacetime $(M, \tilde{g})$ in the proof of theorem 6

Remark 4. This result is sharp in the sense that causal continuity can not replace stable causality in the statement of the theorem. Indeed, the $1+1$ spacetime $\mathbb{R} \times S^{1}$ of coordinates $(t, \theta), \theta \in[0,2]$, metric $\mathrm{d} s^{2}=-\mathrm{d} t^{2}+\mathrm{d} \theta^{2}$ with the timelike segment $\theta=1,0 \leq t \leq 1$, removed does not have lightlike lines, is chronological, and thus stably causal ( $t$ is a time function) but it is not reflective and hence it is not causally continuous. Analogously, chronology can not be weakened to non-total viciousness indeed, for instance, the spacetime of figure 5 is non-totally vicious, does not have lightlike lines but is not even chronological. Nevertheless, it is possible to relax slightly the chronology condition by asking, for instance, that the chronology violating set be confined in a compact or even more weakly to have a compact boundary (see the next section).

Recall that a time function $t: M \rightarrow \mathbb{R}$ is a continuous function which increases on every causal curves, that is, if $\gamma: B \rightarrow M$ is a causal curve, $b_{1}<b_{2}$ implies $t\left(\gamma\left(b_{1}\right)\right)<t\left(\gamma\left(b_{2}\right)\right)$. Hawking proved, improving previous results by Geroch [7], 
that stable causality holds if and only if the spacetime admits a time function (for the direction, time function $\Rightarrow$ stable causality, see [9], for the other direction see [11]). Actually the time function can be chosen smooth with timelike gradient 4] (see also 25). Thus a corollary of theorem 6 is

Theorem 7. If $(M, g)$ is chronological and without lightlike lines then it admits a time function (which can be chosen smooth with timelike gradient).

Recall also that if $t$ is a time function then $F_{a}=\{p: t(p)>a\}$ is an open future set and $\dot{F}_{a}=\{p: t(p)=a\}$. In particular, $S_{a}=\dot{F}_{a}$ is an acausal boundary (hence edgeless), that is, $S_{a}$ is a partial Cauchy hypersurface [1].

The great advantage of theorem 7 is that it allows to considerably weaken the causality and boundary conditions underlying most singularity theorems. Indeed, most of them assume some of the following: (a) global hyperbolicity, (b) a partial Cauchy hypersurface (c) a compact achronal edgeless set (d) a trapped set. Often these global assumptions are made without any further justification, in fact Senovilla in his review [26, p. 803-8] expressed the opinion that these boundary assumptions may represent the main weak point of singularity theorems. Fortunately, theorem [6] justifies the presence of a foliation of partial Cauchy hypersurfaces and hence may be used to weaken the global assumptions made in singularity theorems.

4.1. Absence of lightlike rays. In this section I am going to consider the implications of the absence of lightlike rays. Recall that a future ray is a futureinextendible causal curve which is achronal. Past rays are defined analogously. Chosen a point $c \in(a, b)$ in a lightlike line $\gamma:(a, b) \rightarrow M$, the portion $\left.\gamma\right|_{[c, b)}$ is a lightlike future ray while $\left.\gamma\right|_{(a, c]}$ is a lightlike past ray, thus

Lemma 5. The absence of lightlike future (or past) rays implies the absence of lightlike lines.

Thus, assuming the absence of lightlike future rays one expects to obtain a stronger property than stable causality. Indeed, we have (see also the related result [29, Prop. 4])

Theorem 8. If $(M, g)$ is chronological and without future lightlike rays then it is globally hyperbolic (and the only TIP is M). An analogous past version also holds.

Proof. Since there are no future rays then there are no lightlike lines and the spacetime is stably causal and admits a time function $t$. Let $p \leq q$, we have to prove that $C=J^{-}(q) \cap J^{+}(p)$ is compact. Take $r \in I^{+}(q)$ so that $a=t(r)>t(q)$, and consider the partial Cauchy surface $S_{a}$. Since $C \subset I^{-}(r)$, all the points in $C$ stay in the past set $P_{a}=\{x: t(x)<a\}$. The set $H^{-}\left(S_{a}\right)$ is generated by future lightlike rays (as $S_{a}$ is edgeless) and since by assumption there is no future lightlike ray, $H^{-}\left(S_{a}\right)$ is empty. Thus $C \subset P_{a} \subset D^{-}\left(S_{a}\right) \subset D\left(S_{a}\right)$, the last set being globally hyperbolic. Note that no causal curve from $p$ can escape $D\left(S_{a}\right)$ and hence $P_{a}$ to return to $q$, as $t$ is a time function. Hence $C=J_{D\left(S_{a}\right)}^{-}(q) \cap J_{D\left(S_{a}\right)}^{+}(p)$ is compact. Finally, $(M, g)$ has no TIP but $M$ because the boundary of any TIP is generated by future lightlike rays. 


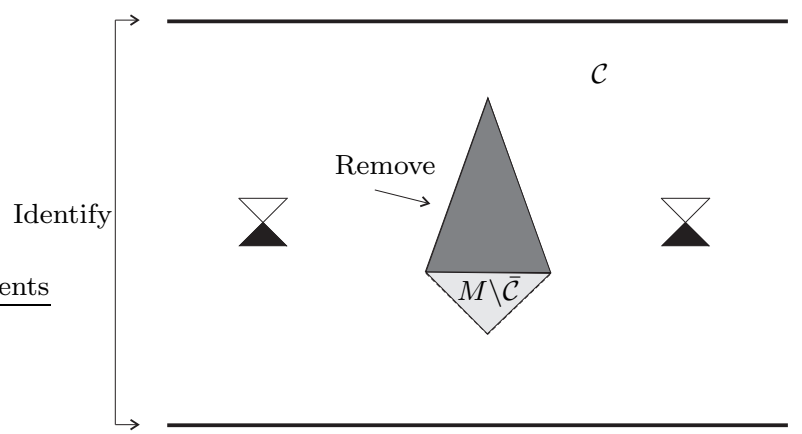

Fig. 5. The figure displays $1+1$ Minkowski spacetime with two spacelike slices identified and a triangle removed. If the angle at the top of the triangle is small enough there are no past lightlike rays.

Note that in theorem 8 chronology can not be weakened to non-total viciousness, i.e. to the condition $\mathcal{C} \neq M$ where $\mathcal{C}$ is the chronology violating set. Indeed, figure 5 gives a counterexample. Nevertheless, if one replaces the absence of $f u$ ture lightlike rays with the absence of lightlike rays then the proof of theorem 12 will show that a non-totally vicious spacetime is chronological (by showing that $\dot{\mathcal{C}}$ if non-empty, contains a lightlike ray), and thus one has:

Theorem 9. If $(M, g)$ is non-totally vicious and without lightlike rays then it is globally hyperbolic (and there are no TIP or TIF but $M$ ).

4.2. Physical considerations. Theorem 8 can be used as a singularity theorem though the null convergence condition is not enough to guarantee that a futurecomplete future-inextendible (affinely parametrized) lightlike geodesic $\gamma:[a,+\infty) \rightarrow$ $M$ admits a pair of conjugate points. A sufficient condition is Tipler's [29, Prop. 1]

$$
\lim _{s \rightarrow+\infty}\left[(s-a) \int_{s}^{+\infty} R_{c d} n^{c} n^{d} \mathrm{~d} s^{\prime}\right]>1
$$

where $n^{c}$ is the tangent vector to $\gamma$ at $\gamma(s)$. Weaker conditions were also considered by Borde [5]. These conditions physically state that the energy density should not drop off too sharply. The assumption is reasonable in those cases where the universe is contracting (or taking the past version, expanding) as one would expect the energy density to increase rather than decrease.

Thus we get the following singularity theorem (past version)

Theorem 10. The following conditions cannot all hold

(i) $(M, g)$ is past null geodesically complete,

(ii) $(M, g)$ is chronological

(iii) $(M, g)$ is non-globally hyperbolic,

(iv) Some energy condition which implies the presence of conjugate points in past-complete past-inextendible lightlike geodesics (e.g.

$$
\lim _{s \rightarrow-\infty}\left[(b-s) \int_{-\infty}^{s} R_{c d} n^{c} n^{d} \mathrm{~d} s^{\prime}\right]>1,
$$


holds on any past-inextendible lightlike geodesic $\gamma:(-\infty, b) \rightarrow M)$.

The nice feature of this theorem is that there is essentially no boundary assumption and the causality conditions are quite weak. There is no assumption on the existence of partial Cauchy surfaces or trapped sets. Of course, the strongest assumption which must be physically justified is made in (iv) but the local expansion of the Universe together with the cosmic background radiation, seem to support it. Then the theorem states, that under the said energy conditions the spacetime is either globally hyperbolic or has singularities. Used in conjunction with Penrose's (1965), and Hawking and Penrose's (1970) singularity theorems [11 it allows to characterize quite precisely what a spacetime looks like if it contains trapped surfaces and it is still null geodesically complete.

We have

Theorem 11. Let $(M, g)$ be a spacetime of dimension greater than 2. If

(i) $(M, g)$ is null geodesically complete,

(ii) $(M, g)$ is chronological,

(ii) There is a future trapped surface,

(iv) The timelike convergence, the generic condition, together with some energy condition which implies the presence of conjugate points in past-complete pastinextendible lightlike geodesics (e.g.

$$
\lim _{s \rightarrow-\infty}\left[(b-s) \int_{-\infty}^{s} R_{c d} n^{c} n^{d} \mathrm{~d} s^{\prime}\right]>1,
$$

holds on any past-inextendible lightlike geodesic $\gamma:(-\infty, b) \rightarrow M)$.

then the spacetime is globally hyperbolic with compact space slices and has a incomplete timelike line.

Proof. The conditions (i), (ii) and (iv) imply (v): the spacetime is globally hyperbolic (theorem 10). The Cauchy hypersurfaces are either compact or noncompact. In the latter case (iii) and (v) imply, by the Penrose singularity theorem, that the spacetime is null geodesically incomplete. Thus (vi): the Cauchy hypersurfaces are compact. The proof of the Hawking-Penrose theorem implies that (i), (ii) or (vi), and (iv) imply that there is a incomplete timelike line.

Since the existence of trapped surfaces is a quite natural consequence of general relativity if matter concentrate enough, theorem 8 supports the global hyperbolicity of the spacetime (and a closed space) provided it is null geodesically complete. Since the conditions are quite reasonable one concludes that the spacetime is either null geodesically incomplete or timelike geodesically incomplete (or both).

Finally I would like to stress that the assumption of null geodesic completeness does not lead to a spacetime picture which contradicts observations. Thus theorems 8 and 6 may have a "positive" role in proving the good causal property of spacetime rather than being used only to prove its singularity. As a matter of fact they can be used to do both (theorem 11). 


\section{The non-chronological case}

So far we have studied the consequence of the absence of lightlike lines under the assumption of chronology. Let us consider the other possibility, namely nonchronological spacetimes. Denote with $\mathcal{C}$ the chronology violating set, with $\mathcal{C}_{\alpha}$, $\mathcal{C}=\bigcup_{\alpha} \mathcal{C}_{\alpha}$, its (open) components and with $B_{\alpha k}$ the (closed) components of the respective boundaries $\dot{\mathcal{C}}_{\alpha}=\bigcup_{k} B_{\alpha k}$.

The next result joins two theorems, one by Kriele [14, Theorem 4] who improved previous results by Tipler [29] and the other by the author [15].

Theorem 12. A non-chronological spacetime without lightlike lines is either totally vicious (i.e. $\mathcal{C}=M$ ) or it has a non-empty chronology violating set $\mathcal{C}$, the

boundaries $\dot{\mathcal{C}}_{\alpha}$ of the components $\mathcal{C}_{\alpha}$, are disjoint and the components $B_{\alpha k}$ of those boundaries are all non-compact. In particular non-totally vicious spacetimes without lightlike lines are non-compact.

For the proof that the sets $\dot{\mathcal{C}}_{\alpha}$ are disjoint I refer the reader to 15 . Instead, I elaborate on Kriele's argument by giving a slightly different proof that the boundaries $B_{\alpha k}$ are non-compact. Indeed, I can give a shorter proof thanks to the limit curve theorem contained in [15] and to the results on totally imprisoned curves contained in [18.

Recall that in the chronology violating set $\mathcal{C}$, Carter's equivalence relation $p \sim q$ iff $p \ll q \ll p$ gives rise to open equivalence classes, moreover, since $\mathcal{C}$ is open, if $x \in \dot{\mathcal{C}}$ it cannot be $x \in \mathcal{C}$. Recall also that with $\Omega_{f}(\eta)$ it is denoted the set $\Omega_{f}(\eta)=\bigcap_{t \in \mathbb{R}} \overline{\eta_{[t,+\infty)}}$ of accumulation points in the future of the causal curve $\eta$, and analogously in the past case. This set is always closed, moreover, it is non-empty iff the curve is partially imprisoned in a compact [18.

Proof. Assume that $B_{\alpha k} \subset \dot{\mathcal{C}}_{\alpha}$ is compact and let $x \in B_{\alpha k}$. Let $x_{n} \in \mathcal{C}_{\alpha}$ such that $x_{n} \rightarrow x$, and let $U \ni x$ be a convex set. There are closed timelike curves $\sigma_{n} \subset \mathcal{C}_{\alpha}$ of starting and ending point $x_{n}$, which are necessarily not entirely contained in $U$ (every convex set is causal). Let $z=x$, then by the limit curve theorem 15] (point 2) there are two cases (corresponding to $0<b<+\infty$, or $b=+\infty$ in that reference).

In the first case there is a closed continuous causal curve $\gamma \in \overline{\mathcal{C}}_{\alpha}$ passing through $x$. It must be achronal since if $p, q \in \gamma, p \ll q$, then $x \leq p \ll q \leq x$ and hence $x \ll x$ which implies $x \in \mathcal{C}$ a contradiction. Thus $\gamma$ is a geodesic with no discontinuity in the tangent vectors at $x$. It can be extended to a lightlike line $\gamma$ by making infinite rounds over $\gamma$ (note that in this case $\Omega_{f}(\gamma)=\Omega_{p}(\gamma)=\gamma$ ).

In the second case there are a future inextendible continuous causal curve $\gamma^{x} \subset \overline{\mathcal{C}}_{\alpha}$ starting at $x$ and a past inextendible continuous causal curve $\gamma^{z} \subset \overline{\mathcal{C}}_{\alpha}$ ending at $x$. If $\gamma^{x} \cap I^{+}(x) \neq \emptyset$ and $\gamma^{z} \cap I^{-}(x) \neq \emptyset$ then for sufficiently large $n$, since $I^{+}$is open, it would be possible to complete a segment of $\gamma_{n}$ to a closed timelike curve passing through $x$ hence $x \in \mathcal{C}$, a contradiction. Thus $\gamma^{x}$ or $\gamma^{z}$, say $\gamma^{x}$, is a lightlike ray. In particular $\gamma^{x}$ being a lightlike ray is achronal and hence can not enter $\mathcal{C}_{\alpha}$, thus $\gamma^{x} \subset B_{\alpha k}$. Now, since $B_{\alpha k}$ is compact and $B_{\alpha k} \cap \mathcal{C}=\emptyset$, results on totally imprisoned causal curves can be applied [18, theorem 3.6]. In particular there is a minimal non-empty closed achronal set $\Omega \subset \Omega_{f}\left(\gamma^{x}\right) \subset B_{\alpha k}$ such that through each point of $\Omega$ there passes one and only one lightlike line, this line is entirely contained in $\Omega$ and for every line $\alpha \subset \Omega$, 
$\Omega_{f}(\alpha)=\Omega_{p}(\alpha)=\Omega$. Just the existence of a lightlike line suffices to conclude the proof that the boundaries $B_{\alpha k}$ are non-compact.

The last statement in a slightly weaker form has been first obtained by Tipler 29, theorem 7]. It follows from the observation that a compact spacetime has a non-empty chronology violating set $\mathcal{C}$ (see [11, Prop. 6.4.2]) thus either $\mathcal{C}=M$ or $\dot{\mathcal{C}}$ is non-empty and compact in contradiction with the absence of lightlike lines.

These results restrict the possible chronology violation in spacetimes without lightlike lines, for instance they state that the chronology violation must extend to infinity. In principle this fact does not mean that a chronology violating region can not develop from regular data. For this to be the case stronger global assumptions than the only absence of lightlike lines should be assumed 29, 13 .

Instead of trying to remove chronology violating sets altogether from the spacetime, it is natural to consider what theorem 6 may say in the cases of chronology violation. The idea is that if $(M, g)$ has a non-empty chronology violating set but $M \neq \overline{\mathcal{C}}$ then the spacetime $\left(N,\left.g\right|_{N}\right)$, where $N$ is any connected components of $M \backslash \overline{\mathcal{C}}$, has empty chronology violating set.

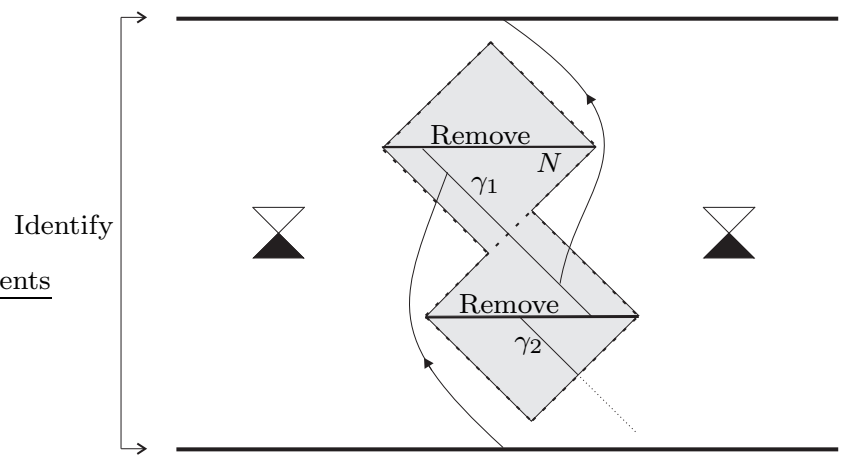

Fig. 6. If $(M, g)$ has a non-empty chronology violating set and has no lightlike line, $\left(N,\left.g\right|_{N}\right)$, with $N$ any component of the shaded region $M \backslash \overline{\mathcal{C}}$, may admit lightlike lines (e.g. the causal curves $\gamma_{1}$ or $\gamma_{2}$ ).

However, even if $(M, g)$ does not have lightlike lines, $\left(N,\left.g\right|_{N}\right)$ may have lightlike lines (see figure 6). This may happen because a lightlike line $\gamma$ for $\left(N,\left.g\right|_{N}\right)$ is not inextendible in $M$, and thus once extended it may enter the chronology violating set (the geodesic $\gamma_{2}$ in the figure). Another possibility is that while $\gamma$ is also inextendible in $M$, the enlargement of the spacetime enlarges the set of timelike curves and hence the possibilities that $\gamma$ is not a line (the geodesic $\gamma_{1}$ in the figure). Thus it is not possible to infer from the absence of lightlike lines for $(M, g)$ the same property for $\left(N,\left.g\right|_{N}\right)$. Actually, neither the converse is true, the Misner spacetime (with region $\mathrm{I}=N$, see figure 32 of [11]) does not have lightlike lines but its analytic extension (I+II) where II is the chronology violating set for I+II, does admit a lightlike line given by the Misner boundary.

There is therefore no immediate way to apply theorem 6 to the non-chronological case apart from that of motivating on physical grounds that some component $N$ does not have lightlike lines. 


\section{Conclusions}

A proof has been given that chronological spacetimes without lightlike lines are stably causal, and that non-totally vicious spacetimes without lightlike rays are globally hyperbolic (together with some other variations). The properties: (i) chronology, (ii) null convergence condition and (iii) null generic condition, are quite reasonable from a physical point of view, moreover, for our purposes (ii) can be weakened to the averaged null convergence condition. Assuming (i), (ii) and (iii) the result of the title of this work translates into the physical statement that null geodesically complete spacetimes are stably causal and therefore admit a time function. Since the existence of some partial Cauchy surface is assumed in most singularity theorems, this result can be used to weaken the assumptions of those theorems. This result may also prove important when applied to the study of the real Universe. Indeed, let us recall that Hawking's and Hawking and Penrose's theorems [11] suggest the existence of an incomplete causal curve which however could well be timelike. In other words our Universe may perhaps be geodesically null complete but timelike incomplete, in which case the main theorem could be applied in the "positive" way to infer the existence of a time function for the Universe. In fact theorem 11 shows that the assumption of null geodesic completeness leads to consequences that do not contradict physical observations.

The Penrose's singularity theorem seems to go against this conclusion as it predicts null incompleteness in those cases in which trapped surfaces form. It must be remarked, however, that Penrose's theorem assumes the existence of a non-compact Cauchy hypersurface thus (i) it assumes the existence of a time function and hence it cannot be used to dismiss the conclusion that a time function exists and (ii) for spacetimes with compact slices its conclusions do not hold. However, even if the space slices are compact, one can still extract information from the proof of Penrose's theorem [22, theorem 14.61]. The result is that, roughly speaking, black holes do not exist. Trapped surfaces may form and locally they may resemble black holes but the global behavior would be quite different. Indeed, their horizons would finally join and swallow the whole spacetime. Thus, without an "exterior", the "interior" could not be distinguished from a usual spacetime.

In conclusion the theorems of this work can be used physically, either in the "negative" way, to prove the existence of singularities or of chronology violating regions, or in the "positive" way to argue for the existence of a time function or of global hyperbolicity. In either case they shade new light on the existence and role of time at cosmological scales.

\section{Acknowledgments}

This work has been partially supported by GNFM of INDAM and by MIUR under project PRIN 2005 from Università di Camerino.

\section{References}

1. Akolia, G. M., Joshi, P., and Vyas, U.: On almost causality. J. Math. Phys. 22, 1243-1247 (1981) 
2. Beem, J. K.: Conformal changes and geodesic completeness. Commun. Math. Phys. 49, 179-186 (1976)

3. Beem, J. K., Ehrlich, P. E., and Easley, K. L.: Global Lorentzian Geometry. New York: Marcel Dekker Inc. (1996)

4. Bernal, A. N. and Sánchez, M.: Smoothness of time functions and the metric splitting of globally hyperbolic spacetimes. Commun. Math. Phys. 257, 43-50 (2005)

5. Borde, A.: Geodesic focusing, energy conditions and singularities. Class. Quantum Grav. 4, 343-356 (1987)

6. Chicone, C. and Ehrlich, P.: Line integration of Ricci curvature and conjugate points in Lorentzian and Riemannian manifolds. Manuscripta Math. 31, 297-316 (1980)

7. Geroch, R.: Domain of dependence. J. Math. Phys. 11, 437-449 (1970)

8. Geroch, R. and Horowitz, G. T.: Global structure of spacetimes, Cambridge: Cambridge University Press, vol. General relativity: An Einstein centenary survey, pages 212-292 (1979)

9. Hawking, S. W.: The existence of cosmic time functions. Proc. Roy. Soc. London, series A 308, 433-435 (1968)

10. Hawking, S. W.: Chronology protection conjecture. Phys. Rev. D 46, 603-611 (1992)

11. Hawking, S. W. and Ellis, G. F. R.: The Large Scale Structure of Space-Time. Cambridge: Cambridge University Press (1973)

12. Hawking, S. W. and Penrose, R.: The singularities of gravitational collapse and cosmology. Proc. Roy. Soc. Lond. A 314, 529-548 (1970)

13. Krasnikov, S.: No time machines in classical general relativity. Class. Quantum Grav. 19, 41094129 (2002)

14. Kriele, M.: The structure of chronology violating sets with compact closure. Class. Quantum Grav. 6, 1607-1611 (1989)

15. Minguzzi, E.: Limit curve theorems in Lorentzian geometry (2007). Preprint: 0712.3942

16. Minguzzi, E.: The causal ladder and the strength of $K$-causality. I. Class. Quantum Grav. 25, 015009 (2008)

17. Minguzzi, E.: The causal ladder and the strength of $K$-causality. II. Class. Quantum Grav. 25, $015010(2008)$

18. Minguzzi, E.: Non-imprisonment conditions on spacetime (2008). J. Math. Phys. In press. Preprint: 0712.3949

19. Minguzzi, E.: Weak distinction and the optimal definition of causal continuity. Class. Quantum Grav. 25, 075015 (2008)

20. Minguzzi, E. and Sánchez, M.: The causal hierarchy of spacetimes, Zurich: Eur. Math. Soc. Publ. House, vol. H. Baum, D. Alekseevsky (eds.), Recent developments in pseudoRiemannian geometry, of ESI Lect. Math. Phys., pages 299 - 358 (2008). gr-qc/0609119

21. Newman, R. P. A. C.: Black holes without singularities. Gen. Relativ. Gravit. 21, 981-995 (1989)

22. O'Neill, B.: Semi-Riemannian Geometry. San Diego: Academic Press (1983)

23. Penrose, R.: Singularities and time-asymmetry, Cambridge: Cambridge University Press, vol. General relativity: An Einstein centenary survey, pages 581-638 (1979)

24. Seifert, H.: The causal boundary of space-times. Gen. Relativ. Gravit. 1, 247-259 (1971)

25. Seifert, H. J.: Smoothing and extending cosmic time functions. Gen. Relativ. Gravit. 8, 815-831 (1977)

26. Senovilla, J. M. M.: Singularity theorems and their consequences. Gen. Relativ. Gravit. 30, 701-848 (1998)

27. Sorkin, R. D. and Woolgar, E.: A causal order for spacetimes with $C^{0}$ Lorentzian metrics: proof of compactness of the space of causal curves. Class. Quantum Grav. 13, 1971-1993 (1996)

28. Thorne, K.: Closed Timelike Curves, Bristol, England: Institute of Physics Publishing, vol. General Relativity and Gravitation, pages 295-315 (1993)

29. Tipler, F. J.: Singularities and causality violation. Ann. Phys. 108, 1-36 (1977)

30. Tipler, F. J.: General relativity and conjugate ordinary differential equations. J. Diff. Eq. 30, 165-174 (1978)

31. Tipler, F. J.: On the nature of singularities in general relativity. Phys. Rev. D 15, 942-945 (1978)

32. Visser, M.: Lorentzian Wormholes. New York: Springer-Verlag (1996)

33. Woodhouse, N. M. J.: The differentiable and causal structures of space-time. J. Math. Phys. 14, 495-501 (1973) 